\title{
Entwicklungsinterventionen und die Macht lokaler Alltagspraxis - das Beispiel der Waldnutzung in Pakistan
}

\section{Urs Geiser, Zürich}

\section{Einleitung}

Die stark genutzten Wälder Nordwest-Pakistans sind seit gut zwanzig Jahren ein hot-spot bi- und multilateraler Entwicklungsanstrengungen - auch weil sich hier globale Entwicklungsthemen der letzten Jahrzehnte wie etwa Armut in Gebirgen, Bedeutung der Wälder, watersheds oder gender konkret addressieren liessen. Doch trotz intensiver Anstrengungen der internationalen Gebergemeinschaft geht es den Wäldern heute so schlecht wie zuvor - eine in Abschnitt 2 vertiefter zu illustrierende Feststellung.

Dies ist Anlass genug, Fragen an die Bedeutung der Entwicklungszusammenarbeit (EZA) zu stellen. Fragen, die weiter zu gehen haben als eine Evaluation von Einzelprojekten. Denn viele der Interventionen der letzten Jahre wurden intensiv evaluiert, gleichwohl wurde das Kernziel der Erhaltung der Wälder nicht erreicht. Dies legt nahe, die Bedeutung der EZA grundsätzlicher zu erforschen, etwa indem ihre Wirkungsweise als eine Arena sozialer Praxis der Politikformulierung und -umsetzung begriffen wird. Abschnitt 3 skizziert eine solche Zugangsweise, hier tentativ als Geographische Forschung zur Entwicklung als soziale Praxis bezeichnet. Diese analytische Perspektive wird in Abschnitt 4 am Beispiel der Waldnutzung in Pakistan eingesetzt. Hierbei wird deutlich, dass zwischen der Formulierung von Entwicklungspolitiken, deren Konkretisierung in Projekten und ihrer praktischen Übersetzung in die lokale Alltagspraxis grosse Unterschiede bestehen, welche häufig im Spannungsfeld zwischen «Projektwelten» und der Alltagspraxis der Beteiligten begründet liegen (Abschnitt 5).

\section{Waldnutzung und Entwicklungsinterventionen in Nordwest-Pakistan}

Offizielle Statistiken bezeichnen rund 5\% der Landesfläche Pakistans als bewaldet. Ein Grossteil findet sich an den Berghängen des Hindukush-Himalaya im nördlichen Bereich der North-West Frontier Province (NWFP; Fig. 1). Diese Wälder sind stark unter Druck; eine satellitenbildbasierte Erhebung postuliert, dass "[using] relevant growth parameters on demand and supply developments and excluding areas that are inaccessible for any wood supply ( $25 \%$ of the total forest area) the total forest stock that existed in 1995 would be completely consumed sometimes between the years 2015 and 2025" (Kreditanstalt Für Wiederaufbau (KfW) et al. 2000).

Kartiert wurden 53\% der Fläche der Provinz mit einem Waldbestand von rund 680'000 ha.

Waldpflege in Pakistan ist Aufgabe der Provinzverwaltung, insbesondere des NWFP Department of Forests, Fisheries and Wildlife. Gemäss international üblichen Praktiken bilden Forstinventuren die Basis zur Erstellung von Waldwirtschaftsplänen mit Vorgaben für jährliche Holzschlagzyklen. Einzelne Holzschläge werden ausgeschrieben und den ausgewählten Holzschlagunternehmern als Kontrakt übertragen.

Die Entwicklungshilfe begann schon in den 1960er Jahren, Kontakte mit dem Forstamt der NWFP zu knüpfen; Experten der Food and Agriculture Organization (FAO) etwa führten Beamte in neuere Techniken der Forstinventur ein. Eine wesentliche Erweiterung begann um 1980, als je ein deutsches (in Kaghan und Siran) und ein schweizerisches Projekt (in Kalam; Fig. 1) gemäss dem damals dominanten Diskurs Waldaspekte sektoriell oder multisektoriell und direkt in lokalen Kontexten aufgriffen. In den 1990er Jahren erhöhte sich die Zahl der waldbezogenen Projekte um ein Vielfaches.

Aus der Vielfalt dieser Projekte wird im Folgenden das Forest Sector Project (FSP) herausgegriffen, welches um 1995 von der Asiatischen Entwicklungsbank (ADB) mit einem Darlehen von 42.6 Mio. US\$ initiiert wurde, mit niederländischer technischer Hilfe und zusätzlicher Unterstützung durch die Schweiz. Projektziel war eine vollständige Reform der geltenden institutionellen Regelungen von der übergeordneten Waldpolitik zur Waldgesetzgebung und den entsprechenden Ausführungsbestimmungen bis zur Organisationsstruktur des Sektors. Nicht zuletzt sollten die Beziehungen zwischen staatlichem Amt und den Bürgern und Bürgerinnen und ihren zivilgesellschaftlichen Organisationen neu definiert und organisiert werden. Als das Projekt Ende 2003 abschloss, waren all diese Reformen im Prinzip durchgeführt. Eine neue Forstpolitik war formuliert, ein neues Forstgesetz erlassen (Government of the North-West Frontier ProvINCE, GONWFP 2002), verschiedene Ausführungsbestimmungen entworfen und in die Vernehmlassung geschickt und Erfolgsberichte für die internationale Entwicklungsdebatte geschrieben. 


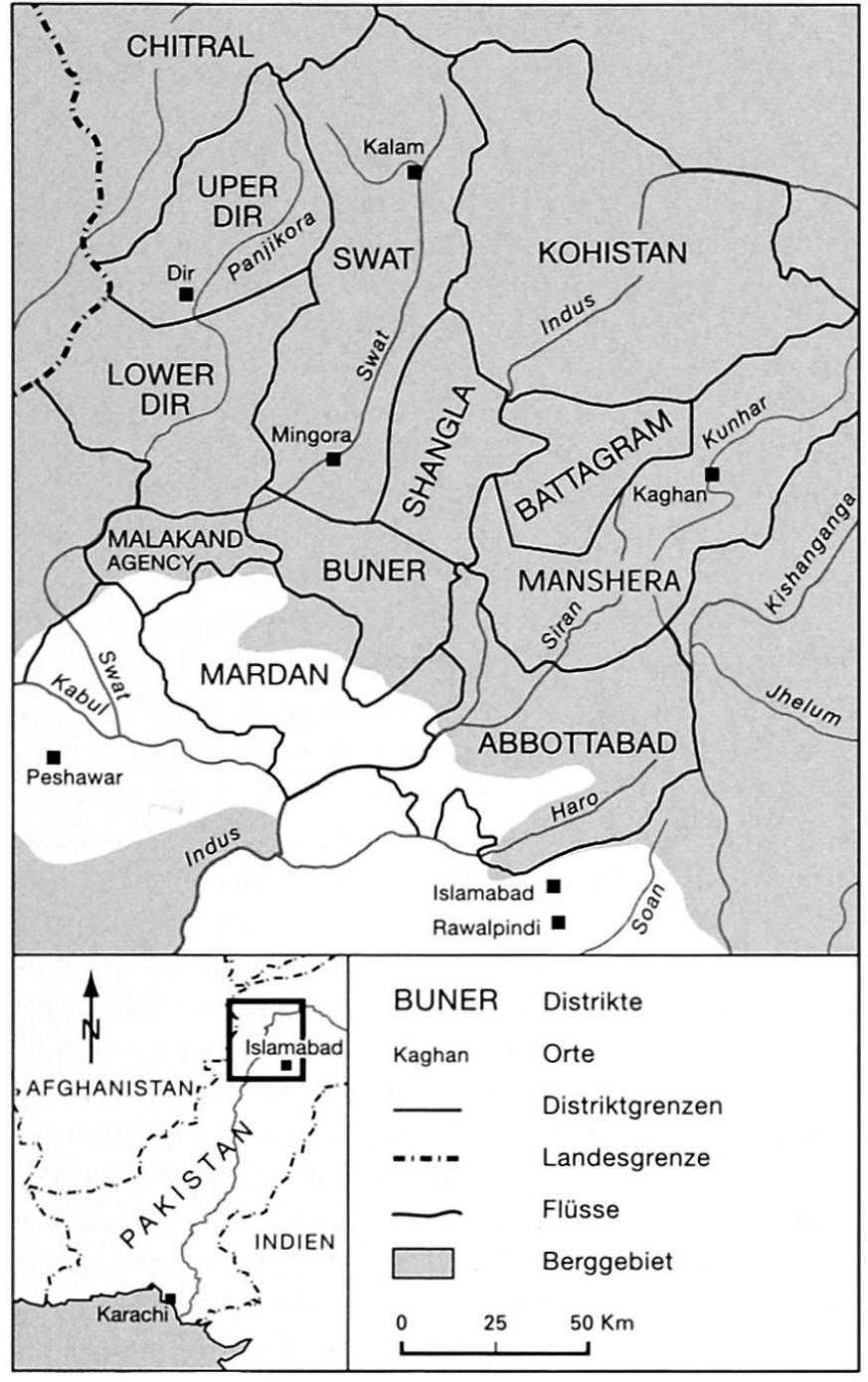

Abb. 1: Die nördlichen Distrikte der North-West Frontier Province (NWFP) Pakistans

The northern districts of the North-West Frontier Province (NWFP) in Pakistan

Les districts au nord de la North-West Frontier Province (NWFP) au Pakistan

Kartengrundlage: eigener Entwurf; Kartographie: M. STEINMANN

Doch für den Wald änderte sich wenig, trotz Forest Sector Project und anderer Interventionen und trotz einer grossen Zahl von internen und externen Evaluationen. Berichte über illegale Entwaldungen erscheinen in der Presse so regelmässig wie vor zwanzig Jahren. Die meisten Angestellten des Forstamtes arbeiten in ihren alten Routinen weiter. Fast alle waldorientierten Projekte endeten in den letzten Jahren, zum Teil aus Frustration. Nachfolgeprojekte sind kaum auszumachen. Es ist wieder ruhiger geworden in den Gängen des Forstamtes.
Natürlich wird die Frage nach den Ursachen der Umsetzungsschwierigkeiten gestellt, auch in Pakistan. Die einen suchen die Schuld bei der Lokalbevölkerung; sie habe nicht aktiver an den Bestrebungen teilgenommen und sei zu stark in ihren traditionellen Ansichten verwurzelt geblieben. Dem Forstamt andererseits wird vorgeworfen, nicht reformfreudig zu sein, kolonial entstandene Strukturen mit ihrem top-down approach nicht zu verändern. Tatsächlich haben sich Abläufe kaum geändert seit der Unabhängigkeit des Landes 1947. Das Personal ist gleich hierarchisch und straff organisiert wie zu Kolonialzeiten (GEISER 2005). Auf lokaler Ebene stellen die foresters die wichtigste Schnittstelle zwischen dem Amt und seinen Regelungen sowie den Wäldern und der Lokalbevölkerung in den abgelegenen Bergtälern des Himalaya-Hindukush dar, haben aber innerhalb der Verwaltung nichts zu sagen. Sie unterstehen den Kreisförstern (forest range officers), diese den divisional forest officers, diese den conservators of forest. Die zentrale Macht aber ist konzentriert in der Provinzhauptstadt Peshawar, beim chief conservator of forests (CCF).

Doch der Vorwurf, dass die Beamten koloniale Arbeitsweisen weiter pflegen, greift zu kurz; die entscheidende Frage ist vielmehr, warum sich alte Routinen auch durch intensive Interventionen im Rahmen einer Vielzahl von Entwicklungsprojekten nur mühsam ändern lassen. Welche Analysezugänge zur Diskussion dieser Frage offeriert die Geographische Entwicklungsforschung?

\section{Anknüpfungspunkte in der Geographischen Entwicklungsforschung}

\subsection{Instrumentelle und kritische Zugänge}

Das intensiv dokumentierte Ende der grossen Entwicklungstheorien (z.B. Booth 1994; Menzel 1992) führte auch in der Geographie zu einer Vielzahl oft parallel laufender Debatten auf der Suche nach detaillierterem Verstehen und teilweise der Formulierung von Erklärungen mittlerer Reichweite (KREUTZMANN 2003 für einen guten Überblick). KRÜGER (2003) bündelt diese Vielfalt in einer ersten Annäherung in drei Bereiche:

(i) Debatten zur Globalisierung,

(ii) Debatten zu Mensch-Umwelt,

(iii) Debatten zu Verwundbarkeit und Lebenssicherung.

Eine zentrale Herausforderung sieht er in der "Überzeugung, dass Anwendungsbezug und entwicklungsstrategische Relevanz der Studien immer im Blickfeld bleiben sollten» (S. 6).

Damit spricht er einen in der Geographischen Entwicklungsforschung heiklen Bereich an, nämlich die 
Beziehung der Forschung zur Praxis. Häufig wird tatsächlich die Forderung gestellt, dass Entwicklungsforschung direkt der Verbesserung der Lebenssituationen in der so genannten Dritten Welt zu dienen hat. Als Ansprechspartner der Forschung gilt - aus naheliegenden Gründen - die Entwicklungszusammenarbeit (EZA) des Nordens. Gemäss Beier \& RenGer (2003: 80f.) wird

«Wissenschaft [...] zu einem strategischen Partner der Entwicklungszusammenarbeit. [...] Entwicklungsprogramme und -projekte sind prädestiniert für eine Flankierung durch Wissenschaftskooperation».

Operationalisiert wird diese Forderung zum Teil schon ex ante auf der Ebene Forschungspolitik, d.h. Eingaben um Gelder für Südforschung müssen Praxisrelevanz vorweisen. Den Forschungsprozess selber versuchen viele mit konkretem Praxisbezug zu verbinden, etwa Begleitforschungen zu EZA-Projekten, gezielte Auftragsforschung (ungern als consultancy bezeichnet), oder die Teilnahme als unabhängige Expertin oder Experte an externen Evaluationen. Auch Regionalentwicklungs- und Forstprojekte in der NWFP gehörten zum Praxisfeld anwendungsorientierter Geographischer Entwicklungsforschung.

Praxisorientierte Forschung ist wichtig und es gilt, sie weiter zu pflegen, doch sie genügt nicht. Gerade das Instrument der Evaluation zeigt die Schwierigkeit, sich mit Entwicklungsmaßnahmen tiefgründiger auseinanderzusetzen. Evaluatoren und Evaluatorinnen stellen sich in den Dienst der Entwicklungspraxis. Sie mögen im besten Fall Korrekturen im Umsetzungprozess der Projektplanungen bewirken oder gar den Abbruch eines Projektes herbeiführen. Sie stellen aber nie das Instrument der Entwicklungsintervention und deren Grundannahmen selber in Frage. Eine solche Beziehung von Forschung und Praxis bezeichnet Mosse (2004: 641) als «instrumentell», und er stellt ihr eine «kritische» gegenüber. Diese unterzieht EZA einer Radikalkritik, sieht in ihr den verlängerten Arm nördlicher Interessen, oder proklamiert sie gar als mitverantwortlich für die Misere des Südens.

Im instrumentellen wie im kritischen Bezug von Forschung zur Praxis aber bleibt die EZA selber eine black box. Die einen versuchen, den Dialog mit der Praxis zu verbessern; die anderen suchen sie im Extremfall zu boykottieren («[development] is not policy to be implemented, but domination to be resisted», MosSE 2004: 643). Genauer hinschauen aber, organisatorische Abläufe und Kommunikation zwischen Beteiligten innerhalb der black box «Entwicklungshilfe» und die Schnittstelle zwischen Projektintentionen und täglicher Lebenswelt der Involvierten thematisieren - dies tun beide nicht. Und genau hier soll der obigen Gliederung von KRÜGER (2003) ein vierter, wenn auch erst in Entstehung begriffener Schwerpunkt Geographischer
Entwicklungsforschung hinzugefügt werden, nämlich (iv) Debatten um Entwicklung als soziale Praxis.

\subsection{Entwicklung als soziale Praxis}

Einem solchen Zugang liegt das Interesse zu Grunde, die Wirkungsweise von Entwicklungsinterventionen unbelastet von einer a priori moralischen Verpflichtung detaillierter auszuloten. Sie versteht EZA als eine Ausprägung gesellschaftlicher Aushandlungsprozesse, neben und mit vielen anderen existierend, eine Ausprägung aber, die durch intensive normative Debatten so aufgeladen ist, dass sie sich einer vertiefteren Auseinandersetzung entzieht.

Eine solche, über die Evaluation oder die Ablehnung des Phänomens EZA hinausgehende Forschung ist im deutschsprachigen Raum anfänglich in der Entwicklungssoziologie auszumachen. Wegbereitend waren Studien von Elwert und Bierschenk in Westafrika. welche Projekte als «soziale Arenen» thematisierten (Elwert \& Bierschenk 1988). Einen Meilenstein stellen die Forschungen von BIERSCHENK und dem französischen Ethnologen Olivier de Sardan zum Phänomen der Entwicklungsmakler dar (AssociaTION EURO-AFRICAINE POUR L'ANTHROPOLOGIE DU CHANGEMENT SOCIAL ET DU DÉvelopPement, APAD 1996; Olivier de Sardan 1995) - Personen, die einerseits in lokalen Kontexten verwurzelt sind und andererseits die Sprache, Denkweise und Handlungsmuster der Entwicklungsprojekte kennen. Dadurch sind sie in der Lage, Projekte gleichsam als Ressource für ihre Netzwerke zu erschliessen. Neuere Arbeiten in der Geographie selber stammen etwa von Korf (2005) oder VÉron et al. (2003). Letztere analysieren im Detail die Interaktionen zwischen Zielgruppen und lokalen Beamten am Beispiel eines Arbeitsprogrammes in West-Bengalen und Bihar.

Am prägnantesten ist die Perspektive im angelsächsischen Raum zu finden, z.B. bei Mosse (2004), WATtS (2001), Lewis, Bebbington (Lewis et al. 2003). Im Zentrum ihres Forschungsinteresses stehen die «complexity of policy as institutional practice, [...] the social life of projects, organizations and professionals and the diversity of interests behind policy models and the perspectives of actors themselves" (MOSSE 2004: 640).

Damit weist Mosse auf ein zentrales Untersuchungsfeld hin, nämlich die Handlungsfähigkeit der Projektbeteiligten und -betroffenen - die Suche also nach einem Verstehen, wie Entwicklung von den Beteiligten gemacht, sozial produziert wird. Eine Hypothese dieser Perspektive von Entwicklung als soziale Praxis ist, dass Einblicke in die Wirkungsweise der EZA durch ein Beleuchten der realen, projektspezifischen Umsetzungsprozesse gewonnen werden können - prägnant, kurz und provokativ von Mosse formuliert als "[but] what, if development practice is not driven by policy?» (Mosse 2004: 640). 


\section{Die Macht lokaler Alltagspraxis}

\subsection{Neue Fragen}

Mit diesem Fokus auf die Alltagswelt nun zurück in die NWFP. Lassen sich Einblicke über das Warum der Umsetzungsschwierigkeiten gewinnen, wenn die alltägliche Realität der Forstprojekte in der NWFP zum Fokus der Forschung wird - nicht deren Absichten und Zielerreichungsgrad? Übersetzt in Forschungsfragen: Was beeinflusst die Lebensunterhaltsstrategien der Forstbeamten und ausländischen Entwicklungsexperten? Welche Rolle spielen darin Bestrebungen von Geberprojekten, eingespielte Routinen zu verändern? Und: Sind es wirklich die Regelungen des Forstamtes, welche den Lebensalltag der Forstfachleute strukturieren?

Diesen Fragen wird im Folgenden am Beispiel des Forest Sector Project nachgegangen. Hierzu wird die Lebenswelt des Projektpersonals entlang eines sozialräumlichen transect vom Zentrum der Macht in Peshawar über regionale Aussenstellen hin zu den Feldbeamten ergründet. Konzeptionell folgt die Schilderung dem Lebensunterhaltsansatz (DEPARTMENT FOR INTERnational Development 2000). Empirische Einsichten stammen aus Feldarbeit in Nordwestpakistan sowie aus Studien, die im Rahmen gemeinsamer wissenschaftlicher Untersuchungen mit pakistanischen Forschenden entstanden sind (Pakistan Research Group 2005).

Die Erforschung konkreter Projektrealität entlang der Perspektive von Entwicklung als soziale Praxis ist methodisch sensitiv. Analysiert wird das konkrete Tun von Menschen im Hier und Jetzt, nicht nur der Lokalbevölkerung, sondern auch von Beamten und Entwicklungsfachleuten. Die Anonymität der Schilderung ist in einem solchen Forschungszusammenhang zwingend. Somit erfolgt die Darstellung der empirischen Ergebnisse im Folgenden narrativ, mit Quellenverweisen, wo möglich und vertretbar.

\subsection{Die Feldbeamten}

Zuerst ins Feld: Die foresters auf der untersten Hierarchiestufe haben diesen Beruf meist nicht aus Liebe zum Wald oder aus Sorge um die Umwelt gewählt. Vielmehr erlaubt er ihnen eine reguläre Staatsstelle mit Aussicht auf eine geregelte, wenn auch kleine Pension. Meist arbeiten sie in ihrer Herkunftsregion, unterstehen also nicht dem Transfersystem. Das heisst auch, dass ihnen keine Unterkunft zur Verfügung gestellt wird, auch keine Transportmittel - obwohl sie für grosse Waldareale zuständig sind (STEIMANN 2004).

Auf der einen Seite wird vom forester erwartet, dass er den Staat vertritt, das Forstgesetz umsetzt. Er ist aber auch Teil des lokalen sozialen Gefüges und eingebun- den in lokale Hierarchien, Machtbeziehungen und Verpflichtungen. Dadurch weiss er, dass der Grossteil der Lokalbevölkerung die staatlichen Waldregelungen nicht akzeptiert. Insbesondere in Distrikten wie Swat, Kohistan oder Dir ist es dem jungen postkolonialen Staat Pakistan noch nicht gelungen, den Menschen ein Gefühl der Staatsbürgerschaft zu vermitteln. Aufgrund historischer Prozesse wurden weite Landstriche erst 1969 formal-rechtlich in den modernen Nationalstaat integriert (SultaN-I-Rome 2005). Dies wirkt sich auch in der Eigentumsordnung bezüglich Wald aus. Für viele gelten weiterhin die überlieferten lokalen Regelungen. Diese sprechen Eigentums- und Nutzungsrechte den alteingesessenen Besitzern von Agrarland zu: wer Acker- oder Weideland sein Eigen nennt, hat damit auch Eigentum am in der Umgebung liegenden Wald. Die Grenzen zwischen so verteilten (und oft umstrittenen) Waldgebieten sind allen bekannt. Später eingewanderte Bevölkerungsgruppen konnten klar spezifizierte, aber eingeschränkte Nutzungsrechte erwerben.

Somit vollzieht der forester seinen Dienst in einer komplexen rechtspluralistischen (BENDA-BECKMANN \& BENDA-BECKMANN 2001) Situation. Als Staatsbeamter vertritt er ein Rechtssystem, das von der Lokalbevölkerung nur selektiv akzeptiert ist, und wo andere Regeln mit anderen Legitimationsbezügen gelten. Hinzu kommt, dass seine formale, vom Staat gebotene Ressourcenausstattung (Lohn, Uniform) sehr klein ist und kaum den Lebensunterhalt zu sichern vermag. Um diese vielschichtige institutionelle und schwierige materielle Situation zu meistern, entwickelten die Feldbeamten Routinen und Praktiken, um ihr setting gleichsam zu «bewirtschaften». Dabei hilft die Verfügungsmacht über informelle Kapitalien: Der forester ist der Beamte in direktem Kontakt mit der Lokalbevölkerung. De jure müsste er viele Angelegenheiten zur Entscheidfällung an seine Vorgesetzten weiterleiten. Doch diese sind weit weg, und der forester entscheidet, was und wie nach oben berichtet wird. Dies erlaubt die flexible Regelung von Vergehen gegen das Waldgesetz oder Anfragen um Bauholz für den lokalen Bedarf.

Die Felderhebungen zeigen, dass diese informellen Ressourcen entscheidend sind für die Sicherung des Lebensunterhaltes. Ein Beispiel: Foresters nehmen sich Freiheiten bei der Umsetzung der staatlichen Gesetze, indem sie deren Aussagen interpretieren und in ihre alltägliche Praxis übersetzen. Sie kennen die geltenden Gesetze durchaus, legen diese aber unterschiedlich aus. Gefragt nach den Regeln, die sie im Falle der Verhaftung eines (aus der Perspektive des staatlichen Gesetzes so bezeichneten) Waldfrevlers zu befolgen haben, geben verschiedene foresters sehr unterschiedliche Antworten. Ähnliches zeigt sich bei der Frage nach der Höhe der Bußen (Tab. 1). 
«Welche Maßnahmen können forest guards und foresters treffen, wenn sie einen Waldfrevler (z.B. bei illegalem Holzschlag) stellen?»

- Ein forest guard kann auf der Stelle eine Buße einziehen, kann dafür aber keinen Beleg ausstellen, weil er kein Belegsbuch hat. Hierzu muss der Täter zum forest range officer gebracht werden.

- Die Buße kann nie an Ort und Stelle eingezogen werden. In jedem Fall ist zuerst ein Bericht zu schreiben und der Täter ist vor Gericht zu bringen.

- Der Täter muss nur vor Gericht gebracht werden, wenn er sich den Beamten widersetzt, oder nicht in der Lage ist, die Buße an Ort und Stelle zu bezahlen.

- Nur foresters und forest range officers dürfen eine Buße an Ort und Stelle einziehen.

- Foresters und forest guards dürfen Täter verhaften.

- Foresters und forest guards dürfen keine Täter verhaften. Dies darf nur von der Polizei gemacht werden.

«Welche Bußen können forest guards und foresters für welches Vergehen im Wald aussprechen (in Rupien pro Kubikfuss)?»

- Himalayazedern (deodar) $400 \mathrm{Rs} / \mathrm{cft}$, Föhren (pine) $150 \mathrm{Rs} / \mathrm{cft}$; dies ist aber auch abhängig vom jeweiligen Marktpreis. Der doppelte Betrag gilt, wenn der Täter in der Nacht gefasst wird.

- Himalayazedern 600 Rs/cft; andere Arten 300 Rs/cft. Der doppelte Betrag gilt, wenn der Täter zum zweiten Mal gefasst wird.

- Die gleiche Buße für alle Baumsorten. $150 \mathrm{Rs} / \mathrm{cft}$ wenn das Holz noch nicht bearbeitet ist; $400 \mathrm{Rs} / \mathrm{cft}$ wenn das Holz bearbeitet ist. Der doppelte Betrag gilt, wenn der Täter in der Nacht gefasst wird.

- $150 \mathrm{Rs} / \mathrm{cft}$ wenn das Holz noch nicht bearbeitet ist; $300 \mathrm{Rs} / \mathrm{cft}$ wenn das Holz bearbeitet ist. Der doppelte Betrag gilt, wenn der Täter in der Nacht gefasst wird.

Tab. 1: Multiple Antworten von Lokalbeamten bezüglich Verfahrensregelungen im Umgang mit Waldfrevlern Multiple answers by local officials regarding rules and regulations in dealing with forest crime

Réponses diverses de fonctionnaires locaux concernant les règles à appliquer en cas d'exploitation délictueuse des forêts

Quelle: STEIMANN 2004: 48

Werden umgekehrt Vertreter der Lokalbevölkerung nach ihren Erfahrungen mit dem «lokalen Staat» gefragt, so äussern einige, dass die Beamten sie nie beim Schlagen von Bäumen für den eigenen Gebrauch hinderten; andere bezahlten - meist gleich vor Ort, ohne nachfolgendes Rechtsverfahren. Andere traten einen Teil des Holzes an den Beamten ab, häufig zwischen einem Drittel und einem Sechstel des gefällten Volumens. Viele bemerkten, dass ein gutes Verhältnis mit den Beamten die Bußen reduzieren hilft. Ein Mann will schon einen Baum im Auftrag des forest guard (Assistent des forester) gefällt haben. Doch an einigen Orten - und dies ist wichtig zu erwähnen und zeigt die Heterogenität lokaler Realität - wurden Personen von lokalen Forstbeamten im Sinne des (staatlichen) Gesetzes angeklagt und vor Gericht gebracht (wo die Fälle allerdings häufig für Jahre pendent sind).

Diese Gespräche wurden anfangs 2003 durchgeführt (Steimann 2004), als das Forest Sector Project schon einige Jahre implementiert war. Doch von diesem Projekt haben die interviewten foresters und forest guards kaum etwas gehört.

\subsection{Die Regionalbehörden}

Die forest range officers sind auf einer höheren Hierarchiestufe angesiedelt, deshalb auch mit mehr Ressourcen ausgestattet: eine Wohnunterkunft mit Büro (oder wenn dies nicht verfügbar ist, eine finanzielle Entschädigung); wenn auch oft in schlechtem Zustand, so sind diese Quartiere doch mit Telephon und Elektrizität ausgestattet. Hinzu kommt ein Fahrzeug. Telephon- und Elektrizitätsrechnungen und auch Benzin oder Diesel für das Fahrzeug sind vom range officer selber zu bezahlen.

Forest range officers sind ebenfalls lokalen Machtstrukturen ausgesetzt, auch wenn sie alle paar Jahre versetzt werden. Dazu gehört zum Beispiel der Vorstand der lokalen Vereinigung der Sägereibesitzer - und auch hier ist Rechtspluralismus auszumachen. Laut staatlichen Regelungen gibt es etwa im Swat-Tal nur wenige Sägereien mit offizieller Genehmigung. Doch es werden Dutzende gefunden, die aus der Sicht des Staates alle illegal operieren. Mittels ihrer zivilgesellschaftlichen Organisation koordinieren sie ihre Interessen und können auch gegenüber dem Vertreter des Forstamtes einflussreicher auftreten. 
Auch wenn das Salär des forest range officer höher ist, so reicht es nicht. Da zum Beispiel in den Orten, in denen sie stationiert sind, oft keine guten Schulen vorhanden sind, lebt die Familie im regionalen Hauptort. Informelle Kapitalien werden auch hier lebenswichtig. Der range officer ist zuständig für die monatliche Auszahlung der Löhne an untere Hierarchiestufen; durch eine Reduktion der Auszahlung lassen sich Telephonoder Dieselrechnungen bezahlen. Auch kontrolliert der range officer die Finanzierung der staatlichen Baumschulen und Aufforstungsarbeiten. Hier ergibt sich Spielraum, z.B. durch das Anpassen von Abrechnungsbelegen, die Verzögerung von Zahlungen an Zulieferer oder Arbeiter. Dem forest range officer obliegt das Anzeichnen der gemäss geltendem Waldwirtschaftsplan jährlich zu fällenden Bäume. Gerade bei dieser Tätigkeit zeigt sich das Spannungsfeld, in dem der Beamte steht, in aller Deutlichkeit. Auf der einen Seite der Waldwirtschaftsplan mit seinen wissenschaftlich hergeleiteten Vorgaben, auf der anderen Seite die Erwartungen der einflussreichen Holzunternehmer. Dazu ist der regionale Holzbedarf enorm und wird durch das Forstamt nicht gedeckt, die (illegalen) Sägereien brauchen Nachschub (diese Situation verschärfte sich gar, als das Forstamt vor ein paar Jahren ein Holzschlagverbot erliess). Geschichten über zuviel angezeichnete Bäume, das Markieren speziell wertvoller oder leicht zugänglicher Bäume und damit verbundene finanzielle Transaktionen gehören zum alltäglichen Gesprächsstoff in den Waldgebieten Nordwest-Pakistans.

Forest range officers sind fest integriert in die Hierarchie des Amtes. So schreibt ihr Vorgesetzter den Annual Confidential Report (ACR) mit der Einschätzung der Leistungen des Försters - ein für die Karriere entscheidendes Papier. Er weiss, dass die Bewertungskriterien im ACR wenig Bezug haben zu seiner täglichen Praxis - auch zu den Ideen, Prozeduren und Experimenten, welche das Forstreformprojekt der Asiatischen Entwicklungsbank (ADB) bringt. Die meisten der interviewten forest range officers berichteten, dass sie von diesen Reformen zwar gehört hätten und manchmal zur Teilnahme an Workshops eingeladen wurden, aber nicht aktiv in die Ausarbeitung des Reforminhaltes involviert waren.

\subsection{Der Entwicklungsexperte}

Verfolgt man den transect zur (von den Wäldern aus gesehen) entfernten Provinzhauptstadt Peshawar, befinden sich dort nebst dem Hauptquartier des Forstamtes auch die Büros des Forest Sector Project mit dessen Vertreter vor Ort, dem chief technical advisor (CTA) aus den Niederlanden. Die Ressourcenausstattung des CTA besteht aus einem sehr guten Salär und vielen zusätzlichen Vorteilen, welche die Position als Entwicklungsexperte mit sich bringt. Im Prinzip wird von ihm erwartet, dass er als change agent vis-à-vis seiner counterparts handelt. Dies sind in aller Regel die Beamten im Forsthauptquartier in Peshawar, dazu einige in den regionalen Zentren. In der Perzeption des CTA betrifft die Feldebene die regionalen Beamten, aber fast nie die lokalen foresters.

Der CTA verwendet Instrumente wie Workshops, Seminare oder gelegentlich PRA (participatory rural appraisal)-Übungen, um seine counterparts von der Notwendigkeit der Reformen zu überzeugen. Einige der Beamten, meist jüngere, versuchen aktiv die an sich als richtig eingeschätzten Ideen in die konkrete Praxis umzuwandeln, z.B. durch die Formierung von Dorfkommittees (SULERI 2002). Gemäss Aussagen von Informanten endeten viele dieser Experimente nach Abbruch des FSP Ende 2003. Für die meisten regionalen Forstbeamten bleiben die Workshops oder PRAs einmalige Ereignisse, gleichsam "Aufführungen» - man übernimmt die neue Sprache in Berichte oder reproduziert sie während den sporadischen Besuchen ausländischer externer Evaluatoren. Man will dem CTA helfen, ihn nicht komprimitieren. In der täglichen Routine aber, ausserhalb dieser projektbezogenen performances, wartet der Zwang der traditionellen forstlichen Verpflichtungen, der macht-vollen lokalen Praxis - und da helfen am besten eingeschliffene Routinen.

Der CTA versucht sein Bestes, institutionellen Wandel zu induzieren. Hierzu ist er auch aufgefordert, mit der «Zivilgesellschaft» zusammen zu arbeiten, sie als Bindeglied zwischen Lokalbevölkerung und dem Forstamt zu propagieren. Hierfür arbeitet er mit einzelnen NGOs: Aus Sicht der meisten Entwicklungsprojekte ist Zivilgesellschaft gleichbedeutend mit der modernen Form nichtstaatlicher Organisationen (NGOs), die oft im Umfeld der Donatoren entstanden sind (GeISER \& MÜLLER-BÖKER 2003). Einige sind aus früheren Entwicklungsprojekten herausgewachsen. Davon gibt es in den Waldgebieten nur sehr wenige, dafür sind sie umso gefragter. Die Vereinigung der Sägereibesitzer (und eine grosse Zahl weiterer, lokal verankerter Organisationen) sind dem CTA unbekannt; seine counterparts weisen ihn auch nicht darauf hin, denn viele dieser Gruppen vertreten (aus der Sicht des Staates) «illegale» Interessen.

Trotz der guten Ressourcenausstattung ist die Anstellungssicherheit des CTA sehr «verwundbar». Er ist Angestellter einer Beratungsfirma aus dem Norden, welche den Kontrakt für das FSP in einem kompetitiven Verfahren vom dortigen Entwicklungshilfeamt zugesprochen erhielt. Natürlich will die Firma den Auftrag nicht verlieren - auch eine Verlängerung des Projektes in eine neue Projektphase wird wieder ausgeschrieben, und die Firma muss sich erneut bewerben. Im vorliegenden Fall verlor die Firma den Auf- 
trag (und der CTA seinen Job) - nicht etwa wegen schlechten Noten durch eine externe Evaluation (eine solche hatte kurz vor Beendigung des Projektes stattgefunden und die Weiterführung empfohlen, wenn auch mit wesentlichen Korrekturen), sondern weil Pakistan plötzlich kein Schwerpunktsland niederländischer Entwicklungshilfe mehr war.

\section{5 «What if development practice is not driven by policy» (Mosse 2004)?}

Das Forest Sector Project griff wesentliche und wichtige Fragen auf. Im Sinne einer instrumentellen (MosSE 2004) Argumentation sind die alten Forstgesetze nicht in der Lage, eine nachhaltige Nutzung des Gutes Wald zu regeln. Das Verhältnis zwischen Verwaltung und Lokalbevölkerung ist konfliktbeladen und muss sich ändern, sollen die Wälder überleben. Die Planung des FSP griff solche Punkte auf, übersetzte sie in Maßnahmen und operationalisierte diese mit Personal, Arbeitsplänen, Geldmitteln, Anreizen. Evaluatoren versuchten die Umsetzung zu beurteilen, Korrekturen vorzuschlagen. Die Entwicklungspraxis gleichsam am Ende des langen Politikumsetzungsprozesses aber entspricht in vielen Teilen nicht den Intentionen. Eine Geographische Forschung zur Entwicklung als soziale Praxis will hierzu keine neue "Grosse Theorie» bieten - aber sie vermag auf einige Warum-Fragen Hinweise zu geben, welche mithalfen, Umsetzungsschwierigkeiten bei waldbezogenen Interventionen in der NWFP zu produzieren.

Die Schilderung zeigt erstens, dass dem Projekt ein Entwicklungsverständnis zugrunde liegt, das als funktionalistisch bezeichnet werden kann: Forstbeamte gelten als legitime Vertreter der Rechtsordnung des modernen Nationalstaates. Ihnen obliegt die Funktion der Umsetzung der Forstgesetze. Die Rechtsordnung an sich (z.B. Forstgesetz) und die Art und Weise ihrer Umsetzung werden von den Donatoren als ungenügend eingestuft. Durch Entwicklungsinterventionen sind diese Schwächen zu beheben und neue Zugänge zu entwickeln. Zentrale Akteure in dieser Entwicklung sind die Vertreter des Staates.

Hier zeigt die Schilderung, zweitens, dass in einer solchen Zugangsweise die Frage nach der Legitimation staatlicher Institutionen (hier Forstgesetz und Forstamt) nicht gestellt wird, aufgrund der internen Logik der Intervention nicht gestellt werden kann. Die Probleme Pakistans, den BürgerInnen ein Gefühl von Staatsbürgerschaft zu vermitteln, und die Feldrealität des Rechtspluralismus weisen aber darauf hin, dass gerade beim Verständnis der Rolle des Staates und seiner Instrumente - und damit auch der Rolle der EZA - grosser Klärungsbedarf besteht.
Drittens schafft der funktionalistische Zugang einen (künstlichen) Unterschied zwischen den mit Entwicklung beauftragten Fachleuten einerseits, und den counterparts und Zielgruppen andererseits. Doch viele der Beteiligten lassen sich nicht so klar bestimmten Rollen zuweisen. Entwicklungsfachleute sind nicht (nur) funktionelles Projektpersonal. Sie sind alle, ob niederländischer CTA oder pakistanischer forest range officer, eingebettet in unterschiedliche soziale Realitäten mit entsprechenden Erwartungen, Normen, Verpflichtungen - und Machtbeziehungen. Das Thematisieren von Akteuren der Entwicklungszusammenarbeit nicht als Externe im Verhältnis zu der zu entwickelnden sozialen Realität, sondern als Teil dieser Realität zeigt eindrücklich, welchen Schwierigkeiten die Involvierten sich im Sichern ihres Lebensalltags ausgesetzt sehen. Hierbei stellt die Entwicklungsmission nur ein institutionelles Feld dar, auf das sie zurückgreifen können. Einige andere Felder sind mächtiger, und gegenüber diesen wirkt Entwicklungszusammenarbeit de facto oft macht-los.

Viertens: Das funktionalistische Entwicklungsverständnis führte im Falle des FSP zur Bildung einer eigenen, in sich sicher stimmigen Projektwelt. Eine eigene Sprache entstand (mehr den internationalen Entwicklungsdiskursen zugewandt), eigene interne Kommunikationen (ein kollegiales Verhältnis zwischen de facto sehr unterschiedlich mächtigen Projektverantwortlichen; das Verfassen von Projektberichten im Sinne der Projektlogik), oder Mechanismen der Rechtfertigung des Tuns (z.B. Evaluationen). Dazu kam eine der Projektwelt angepassste Interpretation lokaler Realitäten, etwa bezüglich Zivilgesellschaft. So lief das FSP Gefahr, für einen Projekterfolg an sich wesentliche Realitäten ausserhalb der eigenen Projektwelt zu übersehen. Die eigentliche Schnittstelle zwischen der projektinternen Realität und der Realität der Menschen vor Ort sind die counterparts - aber die Schwierigkeiten ihrer alltäglichen Lebenswelten hinderten sie oft daran, diese Schnittstellen auch tatsächlich zu erläutern.

Eine Geographische Forschung zur Entwicklung als soziale Praxis mag sich durchaus den Vorwurf gefallen lassen, dass «am Bedarf vorbeigeforscht» wird (BEIER \& RENGER 2003: 81) - sicher am direkten Bedarf der Entwicklungszusammenarbeit. Aber sie vermag aufzuzeigen, dass die Konstruktion und die Operationalisierung von EZA-Projekten Sachverhalte produziert, welche den eigentlichen Zielen dieser Interventionen in vielen Fällen nicht dienen - und dadurch werden Ergebnisse auch solcher Forschung praxisrelevant.

\section{Dank}

Diese Forschung wurde gefördert durch das Swiss National Centre of Competence in Research North- 
South (NCCR N-S), mit finanzieller Unterstützung des Schweizerischen Nationalfonds und der Schweizerischen Direktion für Entwicklung und Zusammenarbeit.

\section{Literatur}

ASSOCIATION EURO-AFRICAINE POUR L'ANTHROPOLOGIE DU CHANGEMENT SOCIAL ET DU DÉVELOPPEMENT (APAD, 1996): Negotiated development: brokers, knowledge, technologies. - Seminarunterlagen des International Seminar of the Euro-African Association for the Anthropology of Social Change and Development (APAD) vom 5.-8.6.1996 in Hohenheim-Stuttgart.

BEIER, C. \& J. RENGER (2003): Entwicklungszusammenarbeit im Wandel - Herausforderungen für den Brükkenschlag von Wissenschaft und Praxis. - In: Petermanns Geographische Mitteilungen 147, 1:74-83.

Benda-Beckmann, F. \& K. von Benda-BeckmanN (2001): Das Forschungsprogramm der Projektgruppe «Rechtspluralismus». - Halle/S.: Max-Planck-Institut für ethnologische Forschung.

Bоотн, D. (1994): Rethinking social development: an overview. - In: Booth, D. (ed.): Rethinking social development. Theory, research and practice. - Essex: Longman Scientific and Technical.

DePaRTMENT For InTERNATIONAL DEVELOPMENT (DFID, 2000): Sustainable livelihoods guidance sheets. - Department for International Development, http://www.livelihood.org/info/info_guidancesheets 10.2.2002.

Elwert, G. \& T. BIERSCHENK (1988): Development aid as an intervention in dynamic systems. - In: Sociologica ruralis 28, 2/3: 99-110.

Geiser, U. (2005): Contested forests in North-West Pakistan. The bureaucracy between the «ecological», the «national», and the realities of a nation's frontier. In: Sivarama krishnan, K. \& G. Cederloef (eds): Ecological nationalism. - New Delhi: Permanent Black.

GEISER, U. \& U. MÜLLER-BÖKER (2003): Gemeinschaft, Zivilgesellschaft und Staat als sozialer Kontext des Lebensalltags in den Bergen Nepals und Pakistans. - In: Jeanneret, F., Wastl-Walter, D., Wiesmann, U. \& M. SchwyN (Hrsg.): Welt der Alpen - Gebirge der Welt. Ressourcen, Akteure, Perspektiven. - Bern, Stuttgart, Wien: Verlag Paul Haupt: 91-104.

Government of the North-West Frontier Province (GoNWFP, 2002): The North-West Frontier Province Forest Ordinance 2002. - Peshawar.

Kreditanstalt für Wiederaufbau (KfW), GesellSCHAFT FÜR ANGEWANDTE FERNERKUNDUNG $\mathrm{mbH}$ (GAF) \& SIGNUM GmbH (2000): Provincial Forest Resource Inventory (PFRI), North West Frontier Province. - Draft final report.

Korf, B. (2005): Die Ordnung der Entwicklung. Zur Ethnographie der Entwicklungspraxis und ihrer ethischen Implikationen. - In: Geographische Zeitschrift 92, 4: 208-226.
Kreutzmann, H. 2003: Theorie und Praxis in der Entwicklungsforschung. Einführung in das Themenheft. - In: Geographica Helvetica 58, 1: 2-10.

KRÜGER, F. (2003): Handlungsorientierte Entwicklungsforschung. Trends, Perspektiven, Defizite. - In: Petermanns Geographische Mitteilungen 147, 1: 6-15.

Lewis, D., Bebbington, A. J., Batterbury, S.P.J., Shah, A., Olson, E., Siddiei, M.S. \& S. Duvall (2003): Practice, power and meaning. Frameworks for studying organisational culture in multi-agency rural development projects. - International working paper 12, London: Centre for Civil Society (CCS), London School of Economics and Political Science.

Menzel, U. (1992): Das Ende der Dritten Welt und das Scheitern der grossen Theorie. - Frankfurt am Main: Suhrkamp.

Mosse, D. (2004): Is good policy unimplementable? Reflections on the ethnography of aid policy and practice. - In: Development and change 35, 4: 639-671.

Olivier De SARDAN, J.-P. (1995): Anthropologie et développement. Essai en socio-anthropologie du changement social. - Marseille: APAD/Paris: Karthala.

Pakistan Research Group (2005): Introduction. http://www.nccr-pakistan.org 15.02.2006.

SteimanN, B. (2004): Decentralization and participation in the forestry sector of NWFP, Pakistan - the role of the state. - NCCR IP6 working paper 7, Zurich.

Suleri, A.Q. (2002): Regional study on forest policy and institutional reforms (RETA). Final report of the Pakistan case study. - Manila: Asian Development Bank.

SULTAN-I-ROME (2005): Forestry in the princely state of Swat and Kalam (North-West Pakistan). A historical perspective on norms and practices. - NCCR IP6 working paper 6 , Zurich.

Véron, R., Corbridge, S., Williams, G. \& M. SRivasTAVA (2003): The everyday state and political society in Eastern India. Structuring access to the employment assurance scheme. - In: The journal of development studies 39, 5: 1-28.

WATTS, M. (2001): Development ethnographies. - In: Ethnography 2, 2:283-300.

\section{Zusammenfassung: Entwicklungsinterventionen und die Macht lokaler Alltagspraxis - das Beispiel der Waldnutzung in Pakistan}

Von Geographischer Entwicklungsforschung wird häufig Praxisrelevanz gefordert, die sich etwa in Begleitforschungen zur Entwicklungszusammenarbeit (EZA) äussert. Solch anwendungsorientierte Forschung ist wichtig, thematisiert aber nie das Instrument der Entwicklungsintervention und deren Grundannahmen selber. Der Artikel argumentiert deshalb für eine Forschung zur Entwicklung als soziale Praxis, mit deren Hilfe die Wirkungsweise der EZA als eine soziale Arena der Politikformulierung und -umsetzung 
begriffen wird. Diese analytische Perspektive wird am Beispiel der stark genutzten Wälder NordwestPakistans eingesetzt. Trotz intensiver Entwicklungsanstrengungen in den letzten zwanzig Jahren geht es den Wäldern heute so schlecht wie zuvor. Durch eine Perspektive von Entwicklung als soziale Praxis wird deutlich, dass zwischen der Formulierung von Entwicklungspolitiken und ihrer praktischen Umsetzung grosse Unterschiede bestehen, welche häufig im Spannungsfeld zwischen «Projektwelten» und der realen Alltagspraxis der Beteiligten begründet liegt.

\section{Summary: Development intervention and dominance of local day-to-day practice exemplified by forestry in Pakistan}

Development studies within geography are expected to be of direct relevance for development projects, for example by providing projects with necessary background information. Such application-oriented research is essential. However, it does not suffice, as it rarely questions the instrument of development interventions as such, including the related underlying assumptions. Therefore, this article argues for a research that focuses on development as social practice. Such research understands development interventions as an arena of societal negotiation. This perspective is exemplified by looking at development interventions in the forestry sector of North-West Pakistan. Here, despite a huge array of donor-supported projects over the last twenty years, forests continue to be under severe pressure. The analysis highlights the huge gap that exists between development policy formulation and its actual translation on the ground. This gap often emerges from the discrepancy between the logics of a "project world», and the complexities of livelihood realities of the involved people.

\section{Résumé: Les projets de développement confrontés aux pratiques quotidiennes. L'exemple de l'exploitation forestière au Pakistan}

On demande fréquemment aux recherches en géographie du développement d'être directement applicables dans le cadre de projets d'aide au développement. De telles recherches sont essentielles, mais elles ne s'interrogent que très rarement sur l'intervention elle-même ou sur les idées qui la sous-tendent. Partant de cette observation, cet article défendra une approche étudiant le développement en tant que pratique sociale. Ce type de recherche considère les interventions d'aide au développement comme une arène sociale dans laquelle se négocient la formulation et la mise en œuvre des politiques. Cette perspective peut être illustrée par l'exemple de l'exploitation forestière dans le nord-ouest du Pakistan où, malgré un grand nombre d'interventions depuis plus de vingt ans, l'état des forêts reste toujours préoccupant. Cette étude analyse le fossé entre la formulation des politiques de développement et leur mise en œuvre sur le terrain. Ce fossé est souvent creusé par les divergences entre le «monde des projets» et les pratiques sociales quotidiennes des populations auxquelles ces projets s'adressent.

Dr. Urs Geiser, Development Study Group, Abteilung Humangeographie, Geographisches Institut, Universität Zürich, Winterthurerstrasse 190, CH-8057 Zürich. e-mail: urs.geiser@geo.unizh.ch

\section{Manuskripteingang/received/manuscrit entré le 3.11.2005}

Annahme zum Druck/accepted for publication/accepté pour l'impression: 3.4 .2006 